\title{
Pilot Study of a Simple Mass Casualty Tracking Application (MCTA) Tested at a High-Throughput Trauma Center
}

\author{
Jason D. Hoskins ${ }^{1 *}$, Ross F. Graham ${ }^{1}$, Matthew Lissauer ${ }^{2}$, Theresa DiNardo² and Les R. Folio ${ }^{3}$ \\ 1 Uniformed Services University of the Health Sciences \\ ${ }^{2}$ University of Maryland Medical Center \\ ${ }^{3}$ National Institutes of Health
}

\begin{abstract}
Background: Efficient and accurate patient tracking during high-throughput situations is critical, especially for resource-limited medicine incurred during natural disasters, terrorist events, chemical threats and other mass casualty situations. Use of air traffic control practices modified to track patients shows promise in military medical applications. We tested a similar application in a busy, level 1 trauma center utilizing a system we call the Mass Casualty Tracking Application (MCTA).
\end{abstract}

Methods: Third party surveyors compared current system to a system using MCTA on sixty four patients in July 2010. Four data points on paired patients were surveyed randomly each hour resulting in 56 total surveys with 50 useable. Written opinion comments were submitted by four staff members exposed to both systems.

Results: Overall, MCTA had no significant difference in errors compared to FirstNet regarding patient data. Hospital staff exposed to both systems preferred FirstNet over MCTA due to familiarity. Hospital staff surveyed for comments on the MCTA indicated that they envisioned the system would be very useful for power outages and/or mass casualty incidents, for determining bottlenecks in patient flow, for tracking patients sent for imaging with useful prompting to seek out imaging read results and as a backup to FirstNet.

Conclusions: MCTA in a busy trauma center is equivalent in its ability to track patients in this specific highthroughput system regarding data correctness/patient location. Though not designed as a replacement to electronic systems, MCTA is capable of performing this task and may be a useful asset for mass casualty events and/or as a backup system due to its "pen and ink" simplicity.

Keywords: Health Information dissemination; Health information systems; Mass casualty incidents; Patient throughput; Air traffic control

Abbreviations: MCTA: Mass Casualty Tracking Application; CT: Computed Tomography; ICU: Intensive Care Unit; ATC: Air Traffic Control; FPS: Flight Progress Strip; PDS: Patient Data Strip; OR: Operating Room; MIST: Mechanism Injury Symptoms Treatment; TRU: Trauma Resuscitation Unit

\section{Introduction}

Efficient and accurate tracking of patients through medical care phases is a critical aspect of any professional medical system. In trauma, this is true from the actual point of injury all the way through definitive care. Military medicine, as conducted in austere environments, continually works to improve tracking methods for patients throughout the medical system of care. In deployed settings, a manual backup system, not dependent on connectivity or electricity, is a requirement since electricity goes out regularly. This holds especially true in the disaster environments seen in the past few years in areas such as Hurricane Katrina in Aug-Sep 2005 and the effects of the tsunami in Japan in Mar 2011.

In recent years, a system based on air traffic control practices designed at the Uniformed Services University in Bethesda was successful at a summer medical field exercise known as Operation Bushmaster at Fort Indiantown Gap, PA [1]. Bushmaster is an intense, four-day capstone field problem for fourth year military medical students which rigorously tests their ability to provide patient care, manage logistics and display leadership in austere day and nighttime operations while still supporting notional combat operations. As a direct result of this test, military medical personnel in $\mathrm{Al} \mathrm{Asad,}$
Iraq learned of the system and requested to put it to the test in the warzone during their monthly mass casualty exercises with the result of significant improvement in their ability to track patients correctly and efficiently compared to their previous methods [2].

There is no known standard (military or civilian) for tracking patients from emergency/trauma rooms through radiology/CT to operating rooms, intensive care unit (ICU), inpatient rooms, or to discharge. Paradoxically, patient safety has become a more visible vulnerability of modern medicine [3]. Basic errors, such as wrong patient identifiers, incorrect site of surgery, or other important missing medical information, has resulted in increased mortality but is preventable with basic safety measures [4]. With this context in mind, the medical profession can benefit from other techniques used in high-risk, complex disciplines, such as aviation, shuttle launch, and nuclear power practices [5]. In fact, a 2007 study proposed using Formula 1 pit stop and aviation models to improve patient throughput from operating room to ICU [6]. For decades, air traffic control (ATC)

*Corresponding author: Jason Hoskins, Uniformed Services University of the Health Sciences, 18 Hopleaf Trail, San Antonio, TX 78256, USA, Tel: 210-2368978; Fax: 210-236-8978; E-mail: hosjd96@gmail.com

Received December 09, 2011; Accepted January 10, 2012; Published January 17,2012

Citation: Hoskins JD, Graham RF, Lissauer M, DiNardo T, Folio LR (2011) Pilot Study of a Simple Mass Casualty Tracking Application (MCTA) Tested at a High-Throughput Trauma Center. J Trauma Treatment 1:108. doi:10.4172/2167-1222.1000108

Copyright: @ 2011 Hoskins JD, et al. This is an open-access article distributed under the terms of the Creative Commons Attribution License, which permits unrestricted use, distribution, and reproduction in any medium, provided the original author and source are credited. 
Citation: Hoskins JD, Graham RF, Lissauer M, DiNardo T, Folio LR (2012) Pilot Study of a Simple Mass Casualty Tracking Application (MCTA) Tested at a High-Throughput Trauma Center. J Trauma Treatment 1:108. doi:10.4172/2167-1222.1000108

Page 2 of 5

has managed the complexities of airspace and aircraft handoff in a simple, tactile, and manual method that has evolved to an efficient and nearly flawless system. Each Flight Progress Strip (FPS) represents an individual aircraft and fits within a strip holder that stacks neatly on strip-holder bays (Figures 1A and 1B). Multiple FPSs are stacked in order of priority within a bay representing a unique stage of flight. The bottom FPS within each section of a bay represents aircraft that have highest priority in that stage of flight. Reprioritization regularly occurs for faster aircraft or those that require expedited throughput for emergency or other priority reasons (e.g. low fuel, ill passenger or weather). This need for constant reprioritization is strikingly similar to the dynamic, iterative triage processes of combat and trauma hospitals. FPSs are also moved from bay to bay (and section to section) as aircraft move from one stage of flight (i.e. airspace) to another.

In an effort to determine the applicability and efficacy in a civilian setting, investigators used the same MCTA system at a busy level 1 state designated trauma center. This particular center handles over 8,000 trauma patients per year. The trauma center receives patients directly from ambulances and from a two+ helicopter-capable helipad and is separate from the emergency room with direct access to the operating rooms (ORs). The trauma center currently uses the Cerner FirstNet
(Cerner Corporation, Kansas City, MO) system to track patients in combination with staff experience, memory and knowledge of patient location and standard procedures. The FirstNet system is updated by administrative clerks at the trauma center desk as official patient data is obtained and usually occurs minutes after the patient arrives in the trauma unit. The data, to include patient bed, trauma identification number and doctor and nurse assigned, is projected onto three plasma screens stationed around the trauma unit. It does not include an inbound or proposed patient location section and has no symbols or methods for tracking patients sent out of the unit for CTs, etc. If these capabilities exist in the system, it is not currently used at this busy trauma unit.

\section{Materials and Methods}

\section{Study design and population}

Approximately six table top trials were conducted from Jan May 2010 in order to study the layout, coordination and tracking requirements specific to this trauma center and to adapt the aluminum-frame MCTA tracking system (Rick Hansen of ATS Global, Silver Spring, MD) to this trauma center's standards/needs. Once the important patient tracking parameters were known, we redesigned
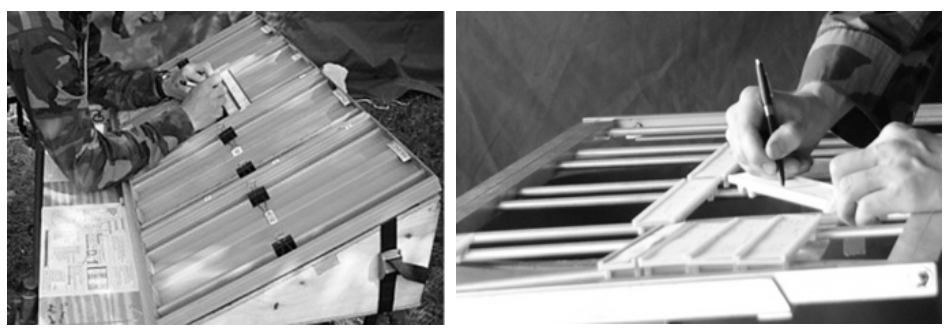

Figure 1: A military medical student updating information to a Patient Data Strip (PDS) during Operation Bushmaster in 2008 (left) and the new aluminum-frame version in 2010 .

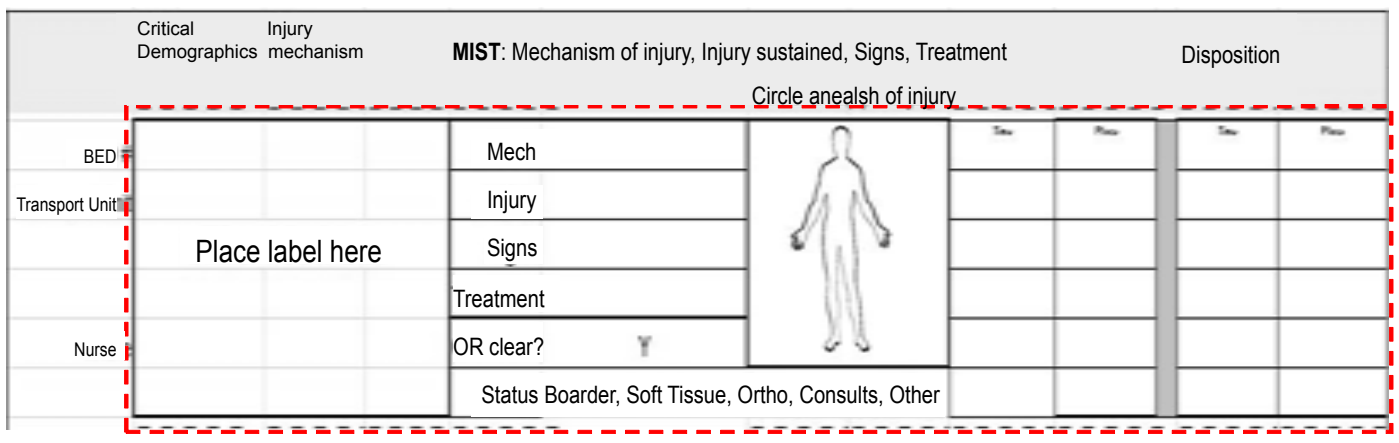

Figure 2: PDS with headings from booklet and blank strip. The area outlined in red is the actual Patient Data Strip that is torn from the PDS Booklet.

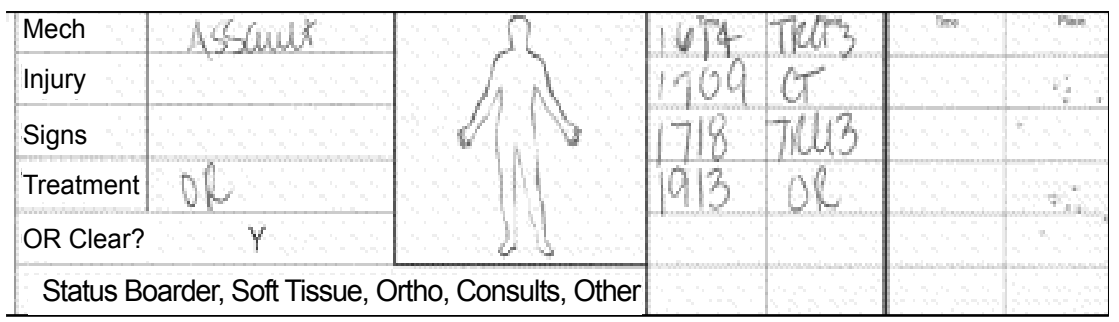

Figure 3: Completed PDS with trauma center label - Actual 5 digit trauma numbers are blocked out. 
a Patient Data Strip (PDS) booklet (courtesy of Yanceyworks, Inc., Sacramento, CA) in June specific to this location's requirements with appropriate PDS markings. The result is in Figures 2 and 3. The formal research trial was conducted over six separate nights between 1-30 Jul 2010 with 24 total hours of patient tracking data obtained. Average patient load on these nights was considered by hospital staff as "light" with very few random "moderate" surges.

Regarding patient injury, we provided a means to track this on the MCTA system using the international MIST format which stands for mechanism, injury, symptoms and treatment [7]. Though not a focus of the trial, it allowed the MCTA tech the opportunity to track this data, if necessary, in order to more distinctly identify a patient in the case of similar names as well as track the specific treatment provided. For a trauma surgeon or czar, this data would also allow for proper triaging and prioritization when necessary. The Cerner FirstNet system has no comparable means of tracking specific MIST parameters; therefore, this data was neither surveyed nor compared.

The PDS Booklet (Figures 4A and 4B) is a spiral bound tablet with approximately 30 total pages and a semi-rigid backing. Each page has three PDSs (one PDS = area outlined in red on Figure 2) lined vertically with perforations to create approximately 90 checkbook style PDSs total that can be easily filled out, removed from the booklet and placed into a stripholder on the bay.

The MCTA is readily modifiable to any hospital or field environment. The final agreement on tracking areas (sections) for the MCTA at this location included "Inbound" patients, "TRU” (Trauma Resuscitation Unit), "Awaiting Imaging", "Imaging Read Pending", "TRU Boarder", "OR Waiting", "OR", "ICU” and "Discharge" (see Table 1 and Figure 5.) "Inbound" included all proposed arrivals not yet at the trauma center, to include inter-hospital transfers and field admissions. The "TRU" section held all PDSs for patients in a trauma center bed unless they were "Awaiting Imaging"/"Imaging Results Pending"/queued for OR ("OR Waiting") (these were placed temporarily in the applicable sections of the MCTA.) The "OR" section held PDSs for patients physically in the OR. The "ICU" section was created to track patients moved from the trauma center to the ICU, but whom the trauma team was still responsible for due to a need for repeat CTs, etc. "Discharge" is self - explanatory, but also includes those patients no longer in trauma center control or responsibility - after discharge or movement to a ward, the PDS was removed from the stripholder and filed.

The population tested included all patients admitted to the trauma center on the testing nights in July 2010. The study was approved with a privacy waiver and waiver of consent by the IRB of the University of Maryland School of Medicine. Patient privacy was maintained with the use of Patient ID tracking numbers as is already assigned using the inplace trauma center system and only rarely were patient names available on the labeling system. The MCTA was kept in a central location of the trauma center, which is itself a semi-secure environment and only those staff with patient care/processing duties were allowed to view the tracking system. The trauma center technician assigned to track patients with the new system constantly monitored the MCTA and entered the initial patient and trauma data as the inbound calls came in. The technician then moved the PDS through the MCTA sections using specific observations of patient movements, specific questions to nursing staff about priorities/plans/status as well as constant situational awareness of the flow of patients garnered by familiarity with the trauma center system. The technician was not allowed to use the FirstNet system to track or verify patient data or during the data surveys and vice versa for the hospital staff using the current FirstNet system of tracking. Only hospital staff/research surveyors/authors of this study were allowed at any time to view the information on the MCTA.

\section{Statistical analysis}

Overall, 64 patients were tracked on the MCTA in those hours with a total of 28 data surveys completed one by one randomly within each hour. Three surveys (covering six patients) were determined unusable

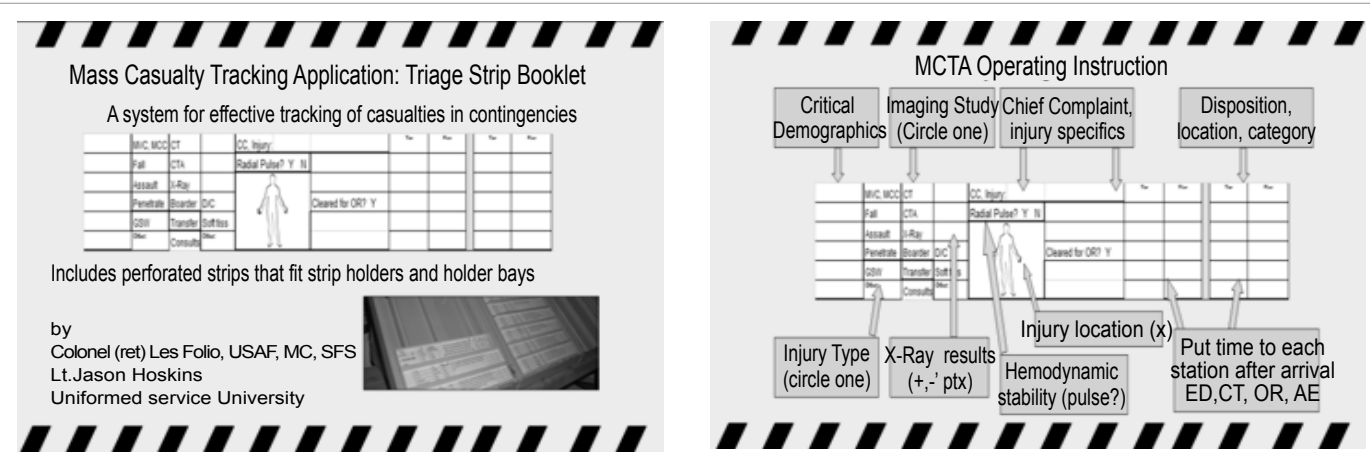

Figure 4: PDS Stripbook - front (left) and back (right) cover that provides readily available pre-perforated strips and basic instructions/guidelines for filling out.

\begin{tabular}{|l|l|}
\hline \multicolumn{1}{|c|}{ Local Term } & Definitio \\
\hline Inbound & Proposed admission from the field or from another hospital \\
\hline TRU & Trauma Resuscitation Unit (trauma center) \\
\hline TRU Boarder & Non-trauma patient physically held in trauma center awaiting placement on non-trauma ward when ward bed becomes available \\
\hline OR Waiting & Queued for OR and placed in priority order \\
\hline ICU & $\begin{array}{l}\text { Intensive Care Unit - not usually used unless patient undergoing repeat imaging and still "owned" by the trauma center who still needs } \\
\text { to track }\end{array}$ \\
\hline
\end{tabular}

Table 1: Table of Local terms for trauma center used in MCTA setup. 


\begin{tabular}{|c|c|c|c|}
\hline & FirstNet (\%correct) & MCTA (\% correct) & Significant Differenc \\
\hline Patient ID & $100(50 / 50)$ & $100(50 / 50)$ & $p=1.0$, no significant difference \\
\hline Patient Nurse & $100(50 / 50)$ & $100(50 / 50)$ & $p=1.0$, no significant difference \\
\hline Pt time arrived & $100(50 / 50)$ & $96(48 / 50)$ & $P=0.1573$, no significant difference \\
\hline Pt current location & $98(49 / 50)$ & $100(50 / 50)$ & $\mathrm{P}=0.3173$, no significant difference \\
\hline Number of total pts in TRU (trauma center) & $84(21 / 25)$ & $96(24 / 25)$ & $P=.1797$, no significant difference \\
\hline $\begin{array}{l}\text { Number of pts for which TRU (trauma center) is currently responsible (in or out } \\
\text { of trauma center) }\end{array}$ & $92(23 / 25)$ & $96(24 / 25)$ & $P=0.5637$, no significant difference \\
\hline Number of inbound field admission & $100(25 / 25)$ & $96(24 / 25)$ & $P=0.3173$, no significant difference \\
\hline Number of inbound transfers from other hospitals & $96(23 / 25)$ & $76(19 / 25)$ & $\mathrm{P}=0.0588$, no significant difference \\
\hline Number of total pts inbound & $96(23 / 25$ & $80(20 / 25)$ & $P=0.1025$, no significant difference \\
\hline
\end{tabular}

Table 2: Summary of McNemar's Test for critical patient tracking data.

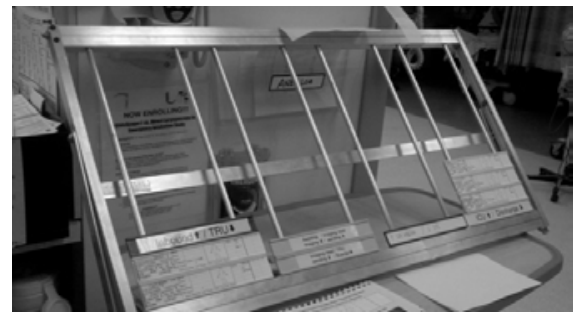

Figure 5: Aluminum-frame MCTA placed on table in central trauma center location.

caused by an inability to survey either the MCTA tech or charge nurse in a timely manner due to high workload. This resulted in 25 total surveys on 50 random patients. McNemar's test (Table 2) was used to determine the error rates of the two systems compared to the correct value as determined by the outside source surveyor. All analyses were done using SAS version 9 (SAS Institute Inc).

\section{Results}

As shown in Table 2, there was no significant difference between FirstNet and MCTA in any specific data point. Additionally, overall results reveal that FirstNet staff was correct for $89 \%$ (335/377) and the MCTA tracker/tech was correct for $91 \%$ (342/377) of all data points. This difference was not statistically significant $(\mathrm{p}=0.327$, McNem r's test).

\section{Discussion}

To the best of our knowledge, this is the first study to test an alternate manual tracking system against a "backbone" electronic system in a level I trauma center. Of note, our system does not require electricity or network availability; providing a dependable backup system during common power and network outages in disaster/ combat. The results show that the MCTA tactile concept of tracking patients is overall equivalent to the FirstNet electronic system in terms of error rate. This indicates that the MCTA system has the same basic capabilities for patient tracking that the more customary FirstNet system provides. Voluntary written opinions on the systems were requested from all staff with exposure or introduction to the MCTA and with past exposure to FirstNet with four hospital staff total providing comments. Overall, these opinions indicate a preference for FirstNet to perform patient tracking. More specifically, comments indicate the main reasons for this include: 1) MCTA requires an additional dedicated body to maintain an updated system (noted in two comments); 2) FirstNet allows staff to see information on patients kept in non-trauma center units; 3) Trauma center staff are already trained and familiar with FirstNet. These may have induced bias towards the existing system that cannot be further tested in this setting.

Conversely, written comments from staff indicated that the MCTA had certain advantages to include: 1) Very useful in the case of power outage or FirstNet outage (noted in 2 comments); 2) Provides a visual method to determine bottlenecks in trauma center patient flow; 3) Provides a method of tracking patients in imaging and prompting for imaging reads; 4) Seen as "great for a field setting"; 5) Viewed as a great "back-up" board. Multidisciplinary team members (researchers, resident physicians, medics in training, etc...) who do not have computer access to FirstNet may also remain aware of rapid patient movement without computer training and access verification procedures.

One staff comment is worth citing in full: "I prefer FirstNet because I am more comfortable with that system. I see that the MCTA can be of great value and eventually better than the FirstNet system due to the ability to track and prioritize patients better. Each system is subject to human errors, but given the same attentiveness from the users, the MCTA has the greater ability to enhance productivity overall.”

Of note, the MCTA was not designed as a replacement for FirstNet, rather it was created to provide a visual contract in a field medical and mass casualty setting with possible positive implications for civilian medical applications. Since it appears that MCTA has the same patient tracking capability with regard to errors, and since it has already proven itself valuable in the military medical setting for mass casualty exercises, then it is safe to suggest that this application should be heavily considered for those applications to include emergency management and disaster medicine. Additionally, both small and large trauma units may consider the MCTA or some equivalent method as a primary or backup system for mass casualty events and as a backup system specifically for power outages or other disaster situations [8]. With this in mind, it is critical that units adopting this strategy begin to incorporate alternate patient tracking sources such as the MCTA into their scheduled mass casualty and disaster preparedness exercises to establish the required critical information and to identify the increased communication flow requirements that occur in these unique situations.

Some limitations of our study need to be recognized. First, we were unable to test the MCTA as a sole-source patient tracker for true standalone evaluation. This was due to the fact that the FirstNet system is used hospital-wide and is a critical system for patient information 
Citation: Hoskins JD, Graham RF, Lissauer M, DiNardo T, Folio LR (2012) Pilot Study of a Simple Mass Casualty Tracking Application (MCTA) Tested at a High-Throughput Trauma Center. J Trauma Treatment 1:108. doi:10.4172/2167-1222.1000108

Page 5 of 5

tracking. Loss of its capabilities and data was considered a degradation of patient care and was never considered. Secondly, we were provided a local trauma medical technician to run the MCTA which enrolls certain biases for/against both systems. This recruitment was critical, however, to using the MCTA in this trial since this person had intimate knowledge of trauma center flow, the FirstNet system and capabilities applied to the trauma center, as well as local terminology, inbound and discharge procedures and local standards of care. We tried to minimize bias by using this technician solely for a specific amount of time after initial train-up on how to use the MCTA. Thirdly, we used two outsidesource surveyors to obtain patient tracking data information. Of the eight nights surveyed, one surveyor obtained data on seven nights and the other surveyor on only one night due to outside scheduling requirements. This resulted in a few instances of differing "data interpretation", specifically with a difference in the treatment and counting of "proposed" inbound patients versus "accepted" inbound patients. Ultimately, we analyzed the data without modifying for this difference and found that there was no overall significant difference in the MCTA versus FirstNet/staff despite this difference in data acquisition/definition.

A major strength of this study includes the method of setup. Researchers spent many hours observing the layout, process and tracking parameters inherent in a level 1 trauma center prior to finalizing the layout and process of the MCTA. In addition, the utilization of a trauma center technician with intimate knowledge of the Cerner FirstNet system, trauma center process and capability to apply this to an MCTA was critical to the successful application of the MCTA to a fast trauma center pace. Third-party surveyors were important to minimize bias when comparing the capabilities of the two systems. Another major strength of the study was utilization of staff that are well versed on the Cerner FirstNet system as the MCTA technician. Though this biases the study somewhat against the MCTA system, it is a strength during testing.

In summary, we demonstrated, to our knowledge for the first time, that a non-electronic-dependent air traffic control-based patient tracking system has an equivalent capability regarding error rate compared to an established electronic patient tracking system such as FirstNet in a level 1 trauma center. Additionally, that a system such as the MCTA has some potentially preferred tracking capabilities not inherent to some electronic systems regarding patient priority, tracking outside of the current unit, and priority for imaging.

\section{Acknowledgements}

Special thanks to Lauren Paul and Hillary Hancock for working as third party surveyors for this project and to Kristen Testerman for learning and working the MCTA system. Thanks to APS Global, LLC (Rick Hansen, Silver Spring MD) for manufacturing/donating the MCTA aluminum-frame tracking board and Yanceyworks, Inc. (Sacramento, CA) for printing and donating the strip books. A final thanks to Dr. Cara Olsen for assistance with the statistics for this project.

\section{References}

1. Hoskins JD, Graham RF, Robinson DR, Lutz CC, Folio LR (2009) Mass Casualty Tracking with Air Traffic Control Methodologies. J Am Coll Surg 208: 1001-1008.

2. Graham RF, Hoskins JD, Cortijo MP, Barbee GA, Folio LR, et al. (2011) A Casualty Tracking System Modeled after Air Traffic Control Methodology Employed in a Combat Support Hospital in Iraq. Mil Med 176: 244-245.

3. Localio AR, Lawthers AG, Brennan TA, Laird NM, Hebert LE, et al. (1991) Relation between malpractice claims and adverse events due to negligence:
Results of the Harvard Medical Practice Study III. N Engl J Med 325: 245-251.

4. Gruen RL, Jurkovich GJ, McIntyre LK, Foy HM, Maier RV (2006) Patterns of errors contributing to trauma mortality: lessons learned from 2,594 deaths. Ann Surg 244: 371-380.

5. Patterson ES, Roth EM, Woods DD, Chow R, Gomes JO (2004) Handoff strategies in settings with high consequences for failure: lessons for health care operations. Int J Qual Health Care 16: 125-132.

6. Catchpole KR, de Leval MR, McEwan A, Pigott N, Elliott MJ, et al. (2007) Patient handover from surgery to intensive care: using Formula 1 pit-stop and aviation models to improve safety and quality. Paediatr Anaesth 17: 470-478.

7. Evans SM, Murray A, Patrick I, Fitzgerald M, Smith S, et al. (2010) Clinica handover in the trauma setting: a qualitative study of paramedics and trauma team members. Qual Saf Health Care 19: e57.

8. Linsenmaier U, Kanz KG, Mutschler W, Pfeifer KJ (2001) [Radiological diagnosis in polytruama: interdisciplinary management]. Rofo 173: 485-493. 